\title{
Nonlocal electrostatics in heterogeneous suspensions using a point-dipole model
}

\author{
Karl von Pfeil and Daniel J. Klingenberga) \\ Department of Chemical and Biological Engineering, University of Wisconsin, 1415 Engineering Drive, \\ Madison, Wisconsin 53706
}

(Received 28 April 2004; accepted 10 June 2004)

\begin{abstract}
The electrostatic dipole moment distribution in heterogeneous suspensions is determined via a self-consistent, point-dipole model, which incorporates nonlocal electrostatics. Predictions agree qualitatively with previous asymptotic results for discontinuous concentration profiles. For small fluctuations in concentration, the dipole strength can be expressed as an expansion in gradients of the concentration. This expansion is incorporated into a linearized continuum model for structure evolution in sheared electrorheological suspensions. Prior stability analysis of a fully local continuum model predicts the formation of concentrated particle stripes oriented in the flow direction, in agreement with experimental observations. Incorporating nonlocal electrostatics suppresses the growth of high wave number fluctuations, providing a more realistic finite rate of growth of fluctuations. Incorporating nonlocal electrostatics in the full nonlinear continuum model produces a single particulate stripe at steady state. (C) 2004 American Institute of Physics.
\end{abstract}

[DOI: $10.1063 / 1.1778483$ ]

\section{INTRODUCTION}

Electrorheological (ER) and magnetorheological (MR) suspensions are well known for their field-dependent rheological properties ${ }^{1-4}$ and are currently being developed for commercial applications. ${ }^{5-7}$ Their unique rheological properties are intimately tied to the heterogeneous structures created by the applied field. Field-induced particle interactions give rise to columnar aggregates in quiescent suspensions. The observed field-dependent yield stress is related to the forces required to distort and break these structures in flow.

Heterogeneous structures persist in shear flow . Inception of shear flow in a suspension with a sufficiently large external field applied in the velocity gradient direction causes the formation of particulate stripes oriented in the flow direction. These stripes consist of bands of high particle concentration separated by bands of low particle concentration. ${ }^{8-12}$

Stripe formation is illustrated in Fig. 1 where photographs of a shear flow experiment with an ER suspension in a concentric cylinder geometry are presented. Here we briefly describe the experiment; more details will be provided in a future publication. The inner cylinder $(25 \mathrm{~mm}$ diameter) is made of stainless steel, and the outer cylinder (27.8 mm diameter) is made of quartz with a thin, transparent, electrically conducting layer deposited on the inner surface. These cylinders are attached to a Bohlin VOR rheometer, in which the inner cylinder is fixed and the outer cylinder is rotated at a constant speed. After shearing the suspension for a short period, an ac electric field $(100 \mathrm{~Hz})$ is applied and the evolution of the suspension is observed visually through the transparent outer cylinder. Photographs for just prior to the application of the electric field and for

\footnotetext{
a) Author to whom correspondence should be addressed; electronic mail: klingen@engr.wisc.edu
}

two later times are presented in Fig. 1 for a 5 vol. \% suspension of $54 \pm 9 \mu \mathrm{m}$ diameter alumina particles in mineral oil (shear rate $\dot{\gamma}=371 / \mathrm{s}$, rms field strength $E=2.0 \mathrm{kV} / \mathrm{mm}$ ). The suspension is homogeneous initially. After the field is applied, concentrated particle stripes oriented in the flow direction begin to form. These structures aggregate and densify slowly with time. As the structure coarsens, the shear stress slowly increases with time.

In previous papers, ${ }^{13,14}$ we presented a continuum model for describing the development of heterogeneous structures in quiescent and sheared ER suspensions. A conservation equation for the particle volume fraction $\phi(\mathbf{x}, t)$ was derived by averaging the mass and momentum balances over the particle phase, wherein the particle flux is related to the particle contribution to the stress. The resulting conservation equation may be expressed as

$$
\frac{\partial \phi}{\partial t}+\langle\mathbf{u}\rangle \cdot \boldsymbol{\nabla} \phi=-\frac{2 a^{2}}{9 \eta_{c}} \boldsymbol{\nabla} \cdot\left[f(\phi) \boldsymbol{\nabla} \cdot \boldsymbol{\sigma}^{(p)}\right],
$$

where $\langle\mathbf{u}\rangle$ is the suspension average velocity, $a$ is the particle radius, $\eta_{c}$ is the continuous phase viscosity, $f(\phi)$ is the sedimentation hindrance function, and $\boldsymbol{\sigma}^{(p)}$ is the particle contribution to the stress. Two contributions to particle stress were accounted for: a hydrodynamic contribution and an electro-
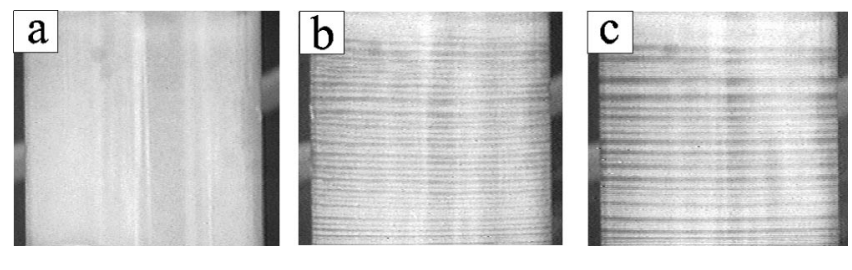

FIG. 1. Photographs of an ER suspension in couette flow between concentric cylinders at different times $t$ after the field has been applied (shear rate $=371 / \mathrm{s}, E=2 \mathrm{kV} / \mathrm{mm}$ at $100 \mathrm{~Hz}$ ). (a) $t=0 \mathrm{~s}$, (b) $t=114 \mathrm{~s}$, (c) $t=264 \mathrm{~s}$. The suspension is a $5 \mathrm{vol} \%$ suspension of alumina particles in mineral oil. 
static contribution. For the hydrodynamic contribution, the model proposed by Morris and Boulay ${ }^{15}$ for sheared, noncolloidal, hard-sphere suspensions was employed,

$$
\boldsymbol{\sigma}^{(p, H)}=-\eta_{n}(\phi) \dot{\gamma} \hat{\mathbf{Q}}+\eta_{p}(\phi)\left[\boldsymbol{\nabla}\langle\mathbf{u}\rangle+\boldsymbol{\nabla}\langle\mathbf{u}\rangle^{T}\right],
$$

where the first term represents the shear-induced normal stresses. Here $\eta_{n}(\phi)$ is the "normal stress viscosity," and $\eta_{p}(\phi)$ is the particle contribution to the shear viscosity [i.e., the suspension shear viscosity is $\left.\eta_{s}(\phi)=\eta_{c}+\eta_{p}(\phi)\right]$. With the $x, y$, and $z$ directions (or the 1,2 , and 3 directions) oriented with the flow, vorticity, and gradient directions, respectively, the normal stresses are specified by the diagonal tensor $\hat{\mathbf{Q}}$ where $\hat{Q}_{\alpha \alpha}=\lambda_{\alpha}$ (no sum) with $\lambda_{1}=1$, and where $\lambda_{2}$ $\cong 0.5$ and $\lambda_{3} \cong 0.8$ provide best fits of various suspension flows. The shear and normal stress viscosities were treated empirically as

$$
\begin{aligned}
& \eta_{p}(\phi)=2.5 \eta_{c} \phi(1-\bar{\phi})^{-1}+K_{s} \eta_{c} \bar{\phi}^{2}(1-\bar{\phi})^{-2}, \\
& \eta_{n}(\phi)=K_{n} \eta_{c} \bar{\phi}^{2}(1-\bar{\phi})^{-2},
\end{aligned}
$$

where $\bar{\phi}=\phi / \phi_{\max }, \phi_{\max }$ is the maximum packing fraction ( $\phi_{\max }=0.68$ herein), and $K_{s}=0.1$ and $K_{n}=0.75$ provided reasonable agreement with experimental data.

For the electrostatic contribution to the stress, the suspension was treated as an isotropic suspension for which the electrostatic particle stress can be written as

$$
\begin{aligned}
\boldsymbol{\sigma}^{(p, E)}= & \epsilon_{0}\left\{\left[\epsilon(\phi)-\epsilon_{c}-\frac{1}{2} a_{1}(\phi)\right] \mathbf{E E}\right. \\
& \left.-\frac{1}{2}\left[\epsilon(\phi)-\epsilon_{c}+a_{2}(\phi)\right] E^{2} \boldsymbol{\delta}\right\},
\end{aligned}
$$

where $\epsilon_{0}=8.8542 \times 10^{-12} \mathrm{~F} / \mathrm{m}$ is the permittivity of free space. A self-consistent mean-field analysis in the pointdipole limit yields a suspension dielectric constant $\epsilon$ and electrostriction coefficients $a_{1}$ and $a_{2}$, given by ${ }^{16}$

$$
\begin{aligned}
& \epsilon(\phi)=\epsilon_{c} \frac{1+2 \beta \phi}{1-\beta \phi}, \\
& a_{1}=0, \\
& a_{2}(\phi)=-\frac{\left[\epsilon(\phi)-\epsilon_{c}\right]\left[\epsilon(\phi)+2 \epsilon_{c}\right]}{3 \epsilon_{c}},
\end{aligned}
$$

where $\epsilon_{c}$ is the relative dielectric constant of the suspending fluid, and $\beta=\left(\sigma_{p}-\sigma_{c}\right) /\left(2 \sigma_{p}+2 \sigma_{c}\right)$ (dc electric fields) or $\left(\epsilon_{p}\right.$ $\left.-\epsilon_{c}\right) /\left(2 \epsilon_{p}+\epsilon_{c}\right)$ (ac electric fields of sufficiently high frequency), where $\sigma_{p}$ and $\sigma_{c}$ are electrical conductivities of the particulate and continuous phases, respectively, and $\epsilon_{p}$ is the relative dielectric constant of the particulate phase.

Linear stability analyses predict the formation of columnar structures in quiescent suspensions. In sheared suspensions, linear stability analysis predicts the formation of stripes, where the concentration profile varies only in the vorticity direction. A simplified, one-dimensional version of the model,

$$
\frac{\partial \phi}{\partial t}=-\frac{2 a^{2}}{9 \eta_{c}} \frac{\partial}{\partial y}\left[f(\phi) \frac{\partial \sigma_{y y}^{(p)}}{\partial y}\right],
$$

was therefore employed for further analysis. Linearizing this conservation equation for small fluctuations in concentration about a mean value, $\phi^{\prime}(y, t) \equiv \phi(y, t)-\phi_{0} \ll 1$, where $\phi_{0}$ is the mean concentration, yields

$$
\frac{\partial \phi^{\prime}}{\partial t}=-M\left[1-\frac{\mathrm{Mn}}{\mathrm{Mn}_{c}\left(\phi_{0}\right)}\right] \frac{\partial^{2} \phi^{\prime}}{\partial y^{2}},
$$

where

$$
\begin{aligned}
& M=\frac{2 a^{2} \epsilon_{0} \epsilon_{c} \beta^{2} E_{0}^{2} f\left(\phi_{0}\right) \phi_{0}}{3 \eta_{c}\left(1-\beta \phi_{0}\right)^{3}}, \\
& \mathrm{Mn}=\frac{\eta_{c} \dot{\gamma}}{2 \epsilon_{0} \epsilon_{c} \beta^{2} E_{0}^{2}}
\end{aligned}
$$

is the Mason number, and

$$
\operatorname{Mn}_{c}=\frac{3 \phi_{\max }^{2}}{4 \lambda_{2} K_{n}} \frac{\left(1-\phi_{0} / \phi_{\max }\right)^{3}}{\left(1-\beta \phi_{0}\right)^{3}}
$$

is the "critical" Mason number. Linear stability analysis shows that stripes should form for $\mathrm{Mn}<\mathrm{Mn}_{c}$. This is qualitatively consistent with our experimental observations with magnetorheological fluids. ${ }^{17}$ However, the stability analysis also predicts the physically unreasonable features of an infinitely fast initial growth rate of stripes with an infinite wave number (i.e., infinitely thin stripes). Thus, the prior continuum modeling effort provides little insight into the important issues of how the structure and rheological properties evolve in time.

The shortcomings described above can be attributed primarily to the local character of the electrostatic stress model employed [Eq. (5)]. The stress, and thus the driving force for particle migration, is assumed to depend only on the concentration at the point of interest. In reality, the stress arises because of electrostatic polarization interactions between particles, which act over considerable distances owing to the $1 / R^{3}$ decay of the disturbance potential generated by a sphere. We thus expect the polarization, and therefore the stress, to depend not only upon the concentration at point but also on the distribution of particles near that point.

In this paper, we develop a self-consistent dipole model for the electrostatic polarization in nonuniform suspensions. We relax the "mean-field" assumption commonly employed in such analyses to obtain a nonlocal model for polarization. The self-consistent field (SCF) model is presented in the following section. In Sec. III A, we compare the predictions of this model for the polarization near a step in concentration with that predicted by an asymptotic analysis presented previously by Keiller and Feuillebois. ${ }^{18}$ The two approaches produce qualitatively similar results, with each approach having its own strengths and weaknesses. We then obtain in Sec. III B a gradient expansion expression for the dipole moment field where the dipole moment magnitude at a point is expressed in terms of gradients of the concentration profile. This expansion is required for predicting the early stages of structure evolution, which is presented in Sec. III C. Here we 
show that incorporation of nonlocal electrostatics produces both a finite initial rate of growth for the fastest growing fluctuations and a finite wave number for these fluctuations. We also show in this section that including nonlocal electrostatics results in a structure consisting of a single stripe at steady state.

\section{SELF-CONSISTENT POINT-DIPOLE MODEL}

In this section we outline how a self-consistent dipole model employed previously for homogeneous suspensions ${ }^{16,19,20}$ can be modified to describe electrostatics in heterogeneous suspensions. Consider a suspension of polarizable spheres in an electric field. The dipole moment of sphere $i$ is given by

$$
\boldsymbol{\mu}_{i}=\alpha \mathbf{E}_{\mathrm{loc}}\left(\mathbf{x}_{i}\right),
$$

where $\alpha=4 \pi \epsilon_{0} \epsilon_{c} a^{3} \beta$ is the particle polarizability. Assuming that the spheres' charge distributions are only dipolar, the local field at the center of sphere $i$ is the macroscopic applied field $\mathbf{E}_{0}$ plus the disturbance dipole fields produced by all of the other spheres,

$$
\mathbf{E}_{\mathrm{loc}}\left(\mathbf{x}_{i}\right)=\mathbf{E}_{0}-\sum_{j \neq i} \mathrm{~T}\left(\mathbf{x}_{i}-\mathbf{x}_{j}\right) \cdot \boldsymbol{\mu}_{j}
$$

where

$$
\mathrm{T}(\mathbf{x})=\frac{1}{4 \pi \epsilon_{0} \epsilon_{c}}\left(\frac{\boldsymbol{\delta}}{|\mathbf{x}|^{3}}-3 \frac{\mathbf{x x}}{|\mathbf{x}|^{5}}\right)
$$

is the dipole interaction tensor.

We wish to determine the ensemble-averaged dipole density given statistical information about the particle positions, namely, the singlet probability density $\mathcal{P}(\mathbf{x})$ and the conditional pair probability density $\mathcal{P}(\mathbf{x} \mid \xi)$. The singlet probability density $\mathcal{P}(\mathbf{x})$ is equated with the position-dependent particle number density $n(\mathbf{x})$.

Averaging the dipole moment of a sphere at $\mathbf{x}_{i}$ over all configurations consistent with the density $n(\mathbf{x})$ gives

$$
\begin{aligned}
\left\langle\boldsymbol{\mu}\left(\mathbf{x}_{i}\right)\right\rangle & =\left\langle\alpha\left[\mathbf{E}_{0}-\sum_{j \neq i} \mathrm{~T}\left(\mathbf{x}_{i}-\mathbf{x}_{j}\right) \cdot \boldsymbol{\mu}\left(\mathbf{x}_{j}\right)\right]\right\rangle \\
& =\alpha\left[\mathbf{E}_{0}-\left\langle\sum_{j \neq i} \mathrm{~T}\left(\mathbf{x}_{i}-\mathbf{x}_{j}\right) \cdot \boldsymbol{\mu}\left(\mathbf{x}_{j}\right)\right\rangle\right] .
\end{aligned}
$$

We equate the average of the sum of all spheres $j \neq i$ with an integral over the pair probability density. The ensembleaveraged dipole density then becomes

$$
\langle\boldsymbol{\mu}(\mathbf{x})\rangle=\alpha\left[\mathbf{E}_{0}-\int_{V} \mathrm{~T}(\mathbf{x}-\boldsymbol{\xi}) \cdot \boldsymbol{\mu}(\boldsymbol{\xi}) \mathcal{P}(\boldsymbol{\xi} \mid \mathbf{x}) d \boldsymbol{\xi}\right] .
$$

As discussed previously, the above integral is conditionally convergent. ${ }^{16,19,20}$ In these references, which addressed uniform suspensions $[n(\mathbf{x})=$ const $]$, the conditional convergence was resolved using a renormalization procedure. The key idea in this procedure is to subtract a second integral from the above equation, whose result is known a priori and which possesses the same conditional convergence behavior. In those cases, the known quantity is the average electric field; since the concentration is uniform, the ensembleaveraged electric field must be equal to the applied macroscopic field $\mathbf{E}_{0}$.

In our case, since $n(\mathbf{x})$ is a function of position, the ensemble-average field may also vary with position and thus is not necessarily known a priori. However, experiments indicate that structures that evolve produce concentration profiles that vary primarily in the direction perpendicular to the field direction. Our prior modeling work ${ }^{13,14}$ produces similar results, with the most rapidly growing fluctuations being those that are independent of $z$ for a field applied in the $z$ direction. We will thus consider here only concentration profiles that depend only on $x$ and $y$, with the macroscopic field applied in the $z$ direction. By symmetry, the resulting ensemble-averaged field for any profile $n(x, y)$ will be constant and equal to the applied macroscopic field $\mathbf{E}_{0}=E_{0} \mathbf{e}_{z}$. We can therefore proceed with the renormalization procedure developed earlier. Below we outline this approach for the new situation where the particle number density is not constant, but depends on $x$ and $y$.

Consider first the ensemble-averaged electric field

$$
\langle\mathbf{E}(\mathbf{x})\rangle=-\int \nabla \Psi \mathcal{P}(C) d C,
$$

where $\int \cdots d C$ represents the integral over all possible configurations consistent with $n(\mathbf{x})$, and $\Psi$ is the total electrostatic potential at $\mathbf{x}$. Following Jeffrey, ${ }^{19}$ we simplify this to leading order in volume fraction by considering only the effect of one sphere, i.e.,

$$
\langle\mathbf{E}(\mathbf{x})\rangle=\mathbf{E}_{0}-\int_{V} \nabla \psi(\mathbf{x}-\boldsymbol{\xi}) \mathcal{P}(\boldsymbol{\xi}) d \boldsymbol{\xi},
$$

where $\psi$ is the single sphere solution for the disturbance potential in a uniform field,

$$
-\nabla \psi(\mathbf{x}-\xi)=\left\{\begin{array}{cc}
-\frac{\mu(\xi)}{4 \pi \epsilon_{0} \epsilon_{c} a^{3}}, & |\mathbf{x}-\boldsymbol{\xi}| \leqslant a \\
-\frac{1}{4 \pi \epsilon_{0} \epsilon_{c}}\left[\frac{\boldsymbol{\delta}}{|\mathbf{x}-\xi|^{3}}-3 \frac{(\mathbf{x}-\boldsymbol{\xi})(\mathbf{x}-\boldsymbol{\xi})}{\mid \mathbf{x}-\xi^{5}}\right] \cdot \boldsymbol{\mu}(\boldsymbol{\xi}), & |\mathbf{x}-\boldsymbol{\xi}|>a .
\end{array}\right.
$$


The integrands in Eqs. (19) and (21) are equivalent as $\mid \mathbf{x}$ $-\boldsymbol{\xi} \mid \rightarrow \infty$ because $\mathcal{P}(\mathbf{x} \mid \boldsymbol{\xi}) \rightarrow \mathcal{P}(\mathbf{x})$ in this limit. Since $\langle\mathbf{E}(\mathbf{x})\rangle$ $=\mathbf{E}_{0}$ for $n(\mathbf{x})=n(x, y)$, the integral in Eq. (21) must be equivalent to zero. Subtracting the integral from Eq. (19), we obtain the following expression for the position-dependent dipole moment in terms of an integral that is absolutely convergent:

$$
\begin{aligned}
\langle\boldsymbol{\mu}(\mathbf{x})\rangle= & \alpha\left\{\mathbf{E}_{0}-\int_{V}[\mathrm{~T}(\mathbf{x}-\boldsymbol{\xi}) \cdot\langle\boldsymbol{\mu}(\boldsymbol{\xi})\rangle \mathcal{P}(\boldsymbol{\xi} \mid \mathbf{x})\right. \\
& -\boldsymbol{\nabla} \psi(\mathbf{x}-\boldsymbol{\xi}) \mathcal{P}(\boldsymbol{\xi})] d \boldsymbol{\xi}\} .
\end{aligned}
$$

We consider here only an idealized, structureless suspension, where the probability density functions are given by $\mathcal{P}(\boldsymbol{\xi})=n(\boldsymbol{\xi})$ and

$$
\mathcal{P}(\boldsymbol{\xi} \mid \mathbf{x})= \begin{cases}0 & \text { for }|\boldsymbol{\xi}-\mathbf{x}| \leqslant 2 a \\ n(\boldsymbol{\xi}) & \text { for }|\boldsymbol{\xi}-\mathbf{x}|>2 a .\end{cases}
$$

Substituting these into Eq. (23) gives the following equation for the self-consistent, ensemble-averaged dipole moment:

$$
\langle\boldsymbol{\mu}(\mathbf{x})\rangle=\alpha\left[\mathbf{E}_{0}+\int_{r<2 a} \boldsymbol{\nabla} \psi(\boldsymbol{\xi}-\mathbf{x}) n(\boldsymbol{\xi}) d \boldsymbol{\xi}\right] .
$$

This equation illustrates the nonlocal character of the polarization, where the dipole at a point depends on an integral of the dipole moments in the neighborhood of the point of interest [the dipole moment depends on the integral via $\nabla \psi$, defined in Eq. (22)].

In the remainder of this paper, we consider concentration profiles that vary only in one direction $y$. Dropping the angled bracket notation, the ensemble-averaged dipole moment magnitude in the $z$ direction, $\mu(y)$, can be expressed as

$$
\mu(y)=\alpha E_{0}+\beta \int_{y-2 a}^{y+2 a} \mu\left(y^{\prime}\right) n\left(y^{\prime}\right) K\left(y^{\prime}-y\right) d y^{\prime},
$$

where

$$
K\left(y^{\prime}-y\right)=\frac{\pi}{2}\left[a^{2}-\frac{1}{4}\left(y^{\prime}-y\right)^{2}\right] .
$$

Equation (26) can be solved numerically, for example, by a Picard iteration. An initial guess for the function $\mu(y)$ is substituted into the integral to obtain an improved approximation for $\mu(y)$. This process is repeated with each successive approximation substituted into the integral until the improved approximation no longer changes. This approach is employed in the following section to determine the dipole moment distribution near a step in concentration. In Sec. III B we describe a gradient expansion solution for $\mu(y)$ valid when the fluctuations in concentration are small.

\section{RESULTS AND DISCUSSION}

\section{A. Comparison with prior work}

Here we consider the effect of nonlocal electrostatics in a nonuniform suspension, where the concentration changes



FIG. 2. Excess polarization as a function of $y$ for a step in concentration using the SCF model and the asymptotic model of Keiller and Feuillebois $(\beta=1)$.

abruptly at a step at $y=0$, where the singlet probability density $\mathcal{P}(y)$ (equal to the number density $n(y)$ ) is given by

$$
\mathcal{P}= \begin{cases}0 & \text { for } y \leqslant 0 \\ n & \text { for } y>0 .\end{cases}
$$

We are interested here in the relationship between the dielectric displacement and electric field for a field applied parallel to the face of the step, $\mathbf{E}_{0}=E_{0} \mathbf{e}_{z}$.

Keiller and Feuillebois ${ }^{18}$ employed Hinch's method ${ }^{21}$ to determine the relationship between the flux and field gradient near such a step change in concentration, including nonlocal effects. Using the notation employed here, they obtained an asymptotic expression to $O\left(\phi^{2}\right)$ for the dielectric displacement,

$$
\mathbf{D}=\epsilon_{0} \epsilon_{c}\left[1+3 \beta \phi^{+}(y)+C_{\|}(y, \beta) \phi^{2}+O\left(\phi^{3}\right)\right] \mathbf{E}_{0},
$$

where $\phi=4 \pi n a^{3} / 3$ is the constant volume fraction within the step and

$$
\phi^{+}(y)=\int_{\left|\mathbf{x}-\mathbf{x}^{\prime}\right|<a} \mathcal{P}\left(y^{\prime}\right) d \mathbf{x}^{\prime} .
$$

The coefficient $C_{\|}(y, \beta)$ is given graphically in Fig. 2 of their paper.

The SCF model [Eq. (26)] is compared to the results obtained by Keiller and Feuillebois in Fig. 2, where the excess polarization is plotted as a function of $y$ for different values of $\phi$. The excess polarization is the particle contribution to the polarization, given by $P^{\mathrm{ex}}=D-\epsilon_{0} \epsilon_{c} E_{0}=n \mu$, where the former form is employed to plot results from the method of Keiller and Feuillebois and the latter form is used to plot results from the SCF model.

The SCF model fails to capture the smooth variation in polarization for $y<0$, primarily because it essentially treats the particles as points. However, the SCF model does capture the variation in polarization for $y>0$. While the results of Keiller and Feuillebois are asymptotically exact as $\phi \rightarrow 0$, their results underpredict the polarization magnitude in the bulk $(y \rightarrow \infty)$ for nonzero $\phi$. The SCF model also predicts a greater range of influence of the interface at large $\phi$, with the polarization varying for larger values of $y$ than that predicted 


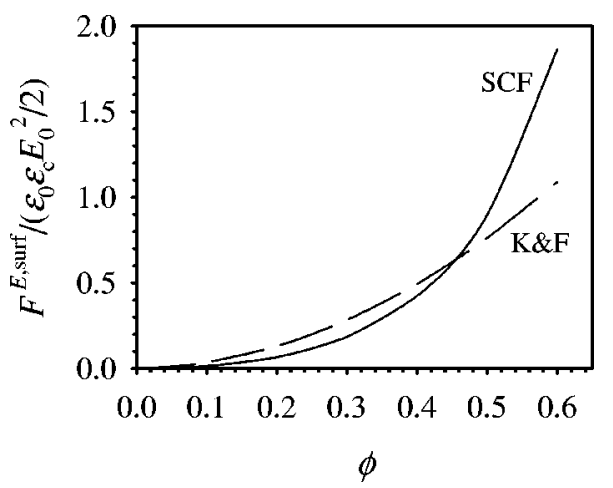

FIG. 3. Surface free energy as a function of concentration for a step in concentration from 0 to $\phi(\beta=1)$.

by Keiller and Feuillebois. Presumably, results predicted by Hinch's method would produce a larger range of influence if carried out to higher order in $\phi$.

The electrostatic contribution to the surface free energy is written as

$$
F^{E \text { surf }}=\int_{-\infty}^{\infty}\left[F^{E}(y)-F^{E, \text { bulk }}\right] d y,
$$

where $F^{E}(y)=-\mathbf{D} \cdot \mathbf{E} / 2$ is the free energy density and $F^{E, \text { bulk }}$ is the free energy density of a uniform suspension with the local concentration,

$$
F^{E, \text { bulk }}= \begin{cases}-\epsilon_{0} \epsilon_{c} E_{0}^{2} / 2 & \text { for } y \leqslant 0 \\ -\mathbf{D}(y \rightarrow \infty) \cdot \mathbf{E}_{0} / 2 & \text { for } y>0 .\end{cases}
$$

The surface free energy for a step in concentration obtained from the SCF model and the method of Keiller and Feuillebois is plotted as a function of particle volume fraction in the step in Fig. 3, for $\beta=1$. Both the models give a positive surface free energy that increases with concentration. The SCF model underpredicts the surface free energy at small concentrations, while the results of Keiller and Feuillebois underpredict the surface free energy at large concentrations.

\section{B. Gradient expansion for $\phi=\phi(x, y)$}

In order to understand how nonlocal contributions to the electrostatics affect the early stages of structure evolution in ER and MR fluids, we must analyze how small fluctuations in concentration affect the polarization. We expand the particle number density at a point $y^{\prime}$ about the the value at $y$,

$$
n\left(y^{\prime}\right)=n(y)+\left(\frac{d n}{d y}\right)_{y}\left(y^{\prime}-y\right)+\frac{1}{2}\left(\frac{d^{2} n}{d y^{2}}\right)_{y}\left(y^{\prime}-y\right)^{2}+\cdots .
$$

Substituting this into Eq. (26), integrating, and gathering like terms, we obtain

$$
\frac{\mu(y)}{\alpha E_{0}}=\tilde{\mu}_{u}(\phi)+K_{1}(\phi) \frac{d^{2} \phi}{d y^{2}}+K_{2}(\phi)\left(\frac{d \phi}{d y}\right)^{2}+\cdots,
$$

where

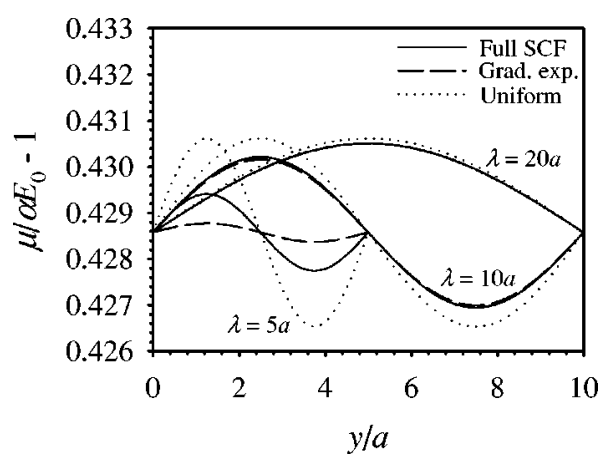

FIG. 4. Dipole moment magnitude as a function of position for sinusoidally varying concentration profiles $(\beta=1)$.

$$
\tilde{\mu}_{u}(\phi)=\frac{1}{1-\beta \phi}
$$

is the solution for the scaled dipole moment magnitude $\mu / \alpha E_{0}$ for a uniform suspension, and

$$
\begin{aligned}
& K_{1}(\phi)=\frac{2}{5} \beta a^{2} \frac{1}{(1-\beta \phi)^{3}}, \\
& K_{2}(\phi)=\frac{4}{5} \beta^{2} a^{2} \frac{1}{(1-\beta \phi)^{4}}
\end{aligned}
$$

describe the influence of spatial derivatives of $\phi$ on the dipole moment.

Equation (34) for $\mu / \alpha E_{0}$, truncated after the $K_{2}$ term, is expected to be valid for relatively small concentration gradients. Consider the behavior of the position-dependent dipole moment for a sinusoidally varying concentration profile of the form

$$
\phi(y)=\phi_{0}+\Delta \phi \sin 2 \pi y / \lambda .
$$

The concentration gradient is proportional to $1 / \lambda$, and thus we expect the gradient expansion to be reasonable for sufficiently large $\lambda$.

In Fig. 4, the dipole moment is plotted as a function of position for three such profiles, with $\phi_{0}=0.3, \Delta \phi=10^{-3}$, and $\lambda=20 a, 10 a$, and $5 a$. The solid curves represent the numerical solution of the full SCF model [Eq. (26)], the dashed curves represent the gradient expansion result, and the dotted curves represent dipole moment magnitude of a uniform suspension with the local value of the concentration [i.e., using Eq. (35) with $\phi=\phi(y)]$. In each case, the dipole magnitude varies sinusoidally with position with the same wavelength as the concentration profile, but with different amplitude (profiles from the full SCF model are not perfectly sinusoidal because of the nonlinear dependence of $\widetilde{\mu}_{u}$ on $\phi$ ). For finite $\lambda$, the amplitude of the dipole moment obtained with the SCF model is smaller than that obtained with the uniform suspension approximation (which is independent of $\lambda$ ). This occurs because the integral in Eq. (26) effectively averages the dipole moment magnitude over the range of two sphere diameters. As $\lambda$ decreases, the moment amplitude obtained from the SCF model decreases because the averaging process en- 


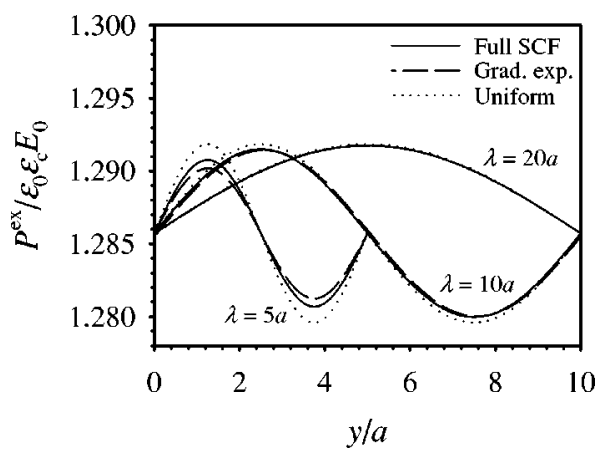

FIG. 5. Excess polarization as a function of position for sinusoidally varying concentration profiles $(\beta=1)$.

compasses a greater fraction of the range of the concentration variation. As $\lambda \rightarrow 0$, the amplitude obtained from the SCF model goes to zero.

The gradient expansion results qualitatively reproduce the behavior of the full SCF model. The amplitude of the dipole moment variation is smaller than that of the uniform suspension approximation. However, the amplitude of the dipole moment variation from the gradient expansion is smaller than that predicted by the full SCF model, more so for smaller $\lambda$.

The excess polarization, $P^{\mathrm{ex}}=n \mu$, is plotted as a function of position in Fig. 5 for the same sinusoidal concentration profiles discussed above. Again, the amplitude of the variation in the polarization obtained from the full SCF model is smaller than that obtained from the uniform suspension approximation. However, the difference in the amplitudes is smaller, and the variation obtained with the gradient expansion more closely follows the SCF model than it does for the dipole moment magnitude. Since the driving force for structure evolution is the free energy, which in turn is more directly relatedto the polarization than the dipole moment magnitude

$\left[F^{E}=-\left(\epsilon_{0} \epsilon_{c} E_{0}^{2}+E_{0} P^{e x}\right) / 2\right.$, see below], the behavior of the predicted polarization is apparently a more critical test of the gradient expansion.

\section{Effect on structure evolution}

In the continuum model described in Sec. I, structure evolution in the one-dimensional model is described by the conservation equation

$$
\frac{\partial \phi}{\partial t}=-\frac{2 a^{2}}{9 \eta_{c}} \frac{\partial}{\partial y}\left[f(\phi) \frac{\partial}{\partial y}\left(\sigma_{y y}^{(p, E)}+\sigma_{y y}^{(p, H)}\right)\right] .
$$

For homogeneous electrified suspensions, the stress is given in terms of the free energy by ${ }^{16,22}$

$$
\sigma_{i k}=\tilde{F} \delta_{i k}+\left.\frac{\partial \tilde{F}}{\partial u_{i k}}\right|_{T, \mathbf{E}}+\frac{1}{2}\left(E_{i} D_{k}+E_{k} D_{i}\right),
$$

where the free energy density is

$$
\tilde{F}=F_{0}-\int_{0}^{\mathbf{E}} \mathbf{D} \cdot d \mathbf{E},
$$

and $F_{0}$ is the free energy density in the absence of an applied field. For the one-dimensional problem, we are interested in only $\sigma_{y y}$. This is obtained from Eq. (40) as

$$
\sigma_{y y}=\widetilde{F}-\phi \frac{\partial \widetilde{F}}{\partial \phi} .
$$

The electrostatic component is simply $\sigma_{y y}^{E}=\sigma_{y y}-\sigma_{y y}\left(E_{0}=0\right)$.

For inhomogeneous suspensions, we assume that we can generalize Eq. (42) to write

$$
\sigma_{y y}^{E}=F^{E}-\phi \frac{\delta \mathcal{F}^{E}}{\delta \phi}
$$

where the total electrostatic free energy $\mathcal{F}^{E}$ is

$$
\mathcal{F}^{E}=\int_{V} F^{E} d V
$$

The electrostatic free energy density is related to the SCF dipole moment magnitude by

$$
\begin{aligned}
F^{E} & =-\frac{1}{2} \mathbf{D} \cdot \mathbf{E} \\
& =-\frac{1}{2}\left(\epsilon_{0} \epsilon_{c} E_{0}^{2}+P^{e x} E_{0}\right) \\
& =-\frac{1}{2} \epsilon_{0} \epsilon_{c} E_{0}^{2}\left[1+3 \beta \phi\left(\frac{\mu}{\alpha E_{0}}\right)\right] .
\end{aligned}
$$

\section{Linear stability analysis}

In order to determine the conditions under which stripes will form from a homogeneous suspension simultaneously subjected to shear and an electric field, we consider the effect of small fluctuations in concentration on the evolution of structure as described by the one-dimensional conservation equation [Eq. (39)]. We let $\phi(y, t)=\phi_{0}+\phi^{\prime}(y, t)$, where $\phi_{0}$ is the average concentration and $\left|\phi^{\prime}(y, t)\right| \ll 1$. Substituting this and the gradient expansion result for $\mu(y) / \alpha E_{0}$ [Eq. (34)] into the expression for the free energy [Eq. (44)], we can obtain the normal stress component $\sigma_{y y}^{E}$ via Eq. (43). Substituting this along with the expression for the hydrodynamic contribution to the normal stress [the $y y$ component of Eq. (2)] into the particle conservation equation [Eq. (39)], we obtain after some simplification

$$
\frac{\partial \phi^{\prime}}{\partial t}=-M\left(1-\frac{\mathrm{Mn}}{\mathrm{Mn}_{c}\left(\phi_{0}\right)}\right) \frac{\partial^{2} \phi^{\prime}}{\partial y^{2}}-M C\left(\phi_{0}\right) \frac{\partial^{4} \phi^{\prime}}{\partial y^{4}},
$$

where $M$ and $\mathrm{Mn}_{c}$ are defined by Eqs. (11) and (13), respectively, and

$$
C\left(\phi_{0}\right)=\frac{a^{2}}{5} \frac{1+\beta \phi_{0}}{1-\beta \phi_{0}} .
$$

To determine the linear stability of a uniform concentration profile, we examine the evolution predicted by Eq. (48) for fluctuations of the form $\phi^{\prime}=e^{i k y} e^{s t}$. Substitution into Eq. (48) yields 


$$
s=M\left(1-\frac{\mathrm{Mn}}{\mathrm{Mn}_{c}}\right) k^{2}-M C k^{4} .
$$

Uniform suspensions will be unstable and stripes will begin to form when $s>0$ for any $k$. Thus the nonlocal electrostatics, which give rise to the term containing $C$, tend to stabilize certain fluctuations. However, the stability boundary $(s=0)$, is still characterized by

$$
\mathrm{Mn}=\mathrm{Mn}_{c},
$$

where the critical Mason number $\mathrm{Mn}_{c}$ is given by Eq. (13). Thus including nonlocal electrostatics does not alter the conditions under which stripes are predicted to form, with stripes still appearing for $\mathrm{Mn}<\operatorname{Mn}_{c}\left(\phi_{0}\right)$.

Although the stability boundary is unaffected, nonlocal electrostatics do alter the fastest growing fluctuations. The wave number that gives the largest value of $s$ is

$$
k_{\max }=\left[\frac{1}{2 C}\left(1-\frac{\mathrm{Mn}}{\mathrm{Mn}_{c}}\right)\right]^{1 / 2}
$$

which corresponds to a growth rate

$$
s_{\max }=\frac{M}{4 C}\left(1-\frac{\mathrm{Mn}}{\mathrm{Mn}_{c}}\right)^{2} .
$$

This differs from that predicted by the previous analysis in which nonlocal electrostatics were ignored, which gave the fastest growing wave number of $k \rightarrow \infty$ with growth rate $s_{\max } \rightarrow \infty$. These quantities are now finite, scaling with the particle size as $k_{\max } \sim 1 / a$ and $s_{\max } \sim 1 / a^{0}$. For typical values of the parameters $\left(\beta=1, \phi_{0}=0.1, \epsilon_{c}=2, E_{0}=10^{6} \mathrm{~V} / \mathrm{m}, \eta_{c}\right.$ $=0.1 \mathrm{~Pa} \mathrm{~s}$ ) with $\mathrm{Mn} \ll \mathrm{Mn}_{c}$, this leads to $s_{\max } \approx 10 \mathrm{~s}^{-1}$, which is consistent with the experimental observation that stripes begin to form rapidly upon the application of a sufficiently large electric field to a sheared suspension.

\section{Steady-state structure}

The structure obtained at steady state after the application of an electric field to a flowing suspension can be obtained by finding the steady-state solution to Eq. (39). In the limit of vanishing shear rates $(\mathrm{Mn} \rightarrow 0)$, the steady state concentration profile will minimize the total electrostatic free energy $\mathcal{F}^{E}$, subject to the constraint $0 \leqslant \phi \leqslant \phi_{\max }$.

A simulated-annealing Monte Carlo algorithm ${ }^{23}$ was employed to find a minimum free energy as follows. Given an initial concentration profile discretized on a periodic domain, a point on the domain is selected at random and the concentration at that point altered randomly (subject to the constraint $\left.0 \leqslant \phi \leqslant \phi_{\max }\right)$. After renormalizing to guarantee $(1 / V) \int_{V} \phi d V=\phi_{0}=$ constant, the total free energy is recalculated. If the free energy is reduced, the step is accepted unconditionally, otherwise it is accepted with probability $p$ $=\exp \left(-\zeta \Delta \mathcal{F}^{E} / \epsilon_{0} \epsilon_{c} E_{0}^{2}\right)$. Using a random concentration fluctuation magnitude of $\Delta \phi=0.004, \zeta$ is slowly increased from $10^{2}$ to $10^{5}$ over the course of $\mathcal{O}\left(10^{7}\right)$ steps. Near the end of this annealing procedure, most steps are rejected, presumably because the free energy is approaching a minimum.

Results from a typical procedure are illustrated in Fig. 6, where the initial and "final" concentration profiles are plotted

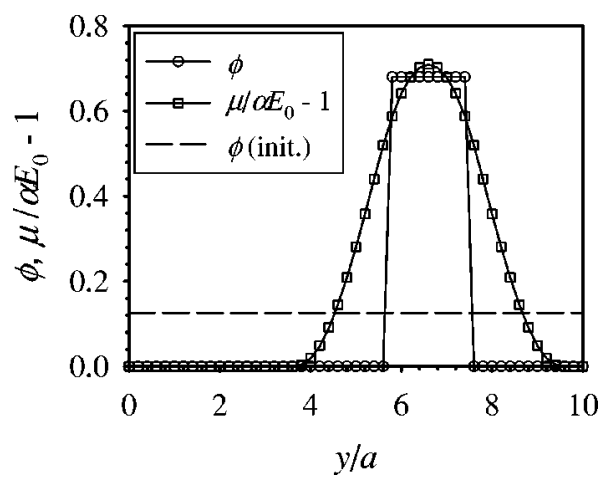

FIG. 6. Initial and final concentration profiles. The final concentration profile was obtained by a Monte Carlo minimization of the free energy. Also shown is the dipole moment magnitude for the final concentration profile.

as a function of position $y$. Also plotted is the dipole moment distribution for the final structure. The initial concentration profile here is a constant $\left[\phi(y)=\phi_{0}=0.3\right]$. The final concentration profile is a stripe-a region of essentially zero concentration and a region of high particle concentration.

Several features of this profile are independent of the initial profile or the sequence of random steps employed. First, although the location of the stripe within the domain may vary, a single stripe always forms. In contrast, the prior continuum model employing only local electrostatics only predicts that stripes will form, but gives no indication of the number. That only a single stripe forms here is expected since each interface gives a positive contribution to the free energy, and thus fewer interfaces are energetically favorable. Second, the concentration profile always varies discontinuously through an interface, varying from $\phi \approx 0$ in the particle-free region to $\phi \lesssim \phi_{\max }$ within the stripe, over a distance of the spatial discretization $\Delta y$. This is consistent with the prior, local continuum model which predicts a discontinuous concentration profile from $\phi=0$ to $\phi_{\max }$ at an interface. We expect that other models for the nonlocal electrostatics will produce similar discontinuous concentration profiles at interfaces.

This steady state structure has several implications for the behavior of sheared ER and MR devices. Since the minimum free energy corresponds to a single stripe, we expect that structures will continue to coarsen until a single stripe is obtained. As illustrated in Fig. 1, coarsening is quite slow, with numerous stripes still present after a considerable period of shearing. Thus steady state will take an even longer time to be reached. Furthermore, the apparent rheological properties will continue to vary as the structure coarsens. We therefore expect that under conditions where stripes form $\left(\mathrm{Mn}<\mathrm{Mn}_{c}\right)$, experiments and devices will essentially always be in unsteady state.

\section{CONCLUSION}

We have illustrated a self-consistent point-dipole model to describe the effect of nonlocal electrostatics on polarization in nonuniform particulate suspensions in the presence of an applied electric field. Analysis was limited to concentration profiles that vary in only one direction perpendicular to a constant applied field. 
Results for a step concentration profile $[\phi(y)=0$ for $y<0, \quad \phi(y)=\phi_{0}$ for $y \geqslant 0$ ] were compared to a prior asymptotic model described by Keiller and Feuillebois. Both approaches produce a polarization profile that increases slowly through $y=0$. The self-consistent method produces a discontinuity that results from treating the particles as point objects. The excess surface free energies obtained from the two methods are qualitatively similar.

The self-consistent polarization model was incorporated in a two-fluid continuum model for the evolution of structure in electrorheological suspensions. Incorporating the nonlocal electrostatics in a linearized version of the transient evolution equation provides a finite growth rate and wave number for the fast growing structures, in contrast to the fully local model which predicts that the fastest growing wave number is infinite, with infinite growth rate. Steady-state structures (in the limit of zero Mason number) were obtained by minimizing the electrostatic free energy. Inclusion of nonlocal electrostatics produces a single particle-rich stripe at steady state, surrounded by particle-free suspending fluid. The concentration within the stripe is uniform.

\section{ACKNOWLEDGMENTS}

The authors thank J. F. Morris and M. D. Graham for many helpful comments, and B. Weber and M. Ward for helping with the experiments. This research was supported in part by the NSF-funded University of Wisconsin Materials Research Science and Engineering Center on Nanostructured Materials and Interfaces, Grant No. DMR-0079983, and by the German Academic Exchange Service (DAAD).
${ }^{1}$ J. Rabinow, AIEE Trans. 67, 1308 (1948).

${ }^{2}$ W. M. Winslow, J. Appl. Phys. 20, 1137 (1949).

${ }^{3}$ J. M. Ginder, in Encyclopedia of Applied Physics, edited by G. L. Trigg (VCH, Weinheim, 1996), Vol. 16, p. 487.

${ }^{4}$ M. Parthasarathy and D. J. Klingenberg, Mater. Sci. Eng., R. 17, 57 (1996).

${ }^{5}$ J. D. Carlson and J. L. Sproston, in Proceedings of the 7th International Conference on New Actuators, edited by H. Borgmann (Messe Bremen $\mathrm{GmbH}$, Bremen, 2000), p. 126.

${ }^{6}$ D. J. Klingenberg, AIChE J. 47, 246 (2001).

${ }^{7}$ J. Ouellette, The Industrial Physicist, 14-17, December 2003/January 2004.

${ }^{8}$ G. Bossis, E. Lemaire, J. Persello, and L. Petit, Prog. Colloid Polym. Sci. 89, 1 (1992).

${ }^{9}$ G. Bossis, Y. Grasselli, E. Lemaire, J. Persello, and L. Petit, Europhys. Lett. 25, 335 (1994).

${ }^{10}$ S. Henley and F. E. Filisko, J. Rheol. 43, 1323 (1999).

${ }^{11}$ O. Volkova, S. Cutillas, and G. Bossis, Phys. Rev. Lett. 82, 233 (1999).

${ }^{12}$ S. Vieira, L. B. Pompeo Neto, and A. C. F. Arruda, J. Rheol. 44, 1139 (2000).

${ }^{13}$ K. von Pfeil, M. D. Graham, D. J. Klingenberg, and J. F. Morris, Phys. Rev. Lett. 88, 188301 (2002).

${ }^{14}$ K. von Pfeil, M. D. Graham, D. J. Klingenberg, and J. F. Morris, J. Appl. Phys. 93, 5769 (2003)

${ }^{15}$ J. F. Morris and F. Boulay, J. Rheol. 43, 1213 (1999).

${ }^{16}$ Y. M. Shkel and D. J. Klingenberg, J. Appl. Phys. 83, 7834 (1998).

${ }^{17}$ J. C. Ulicny, M. A. Golden, C. S. Namuduri, and D. J. Klingenberg J. Rheol. (accepted for publication).

${ }^{18}$ R. A. Keiller and F. Feuillebois, Proc. R. Soc. London, Ser. A 440, 717 (1993).

${ }^{19}$ D. J. Jeffrey, Proc. R. Soc. London, Ser. A 335, 355 (1973).

${ }^{20}$ P. M. Adriani and A. P. Gast, Phys. Fluids 31, 2757 (1988).

${ }^{21}$ E. J. Hinch, J. Fluid Mech. 83, 695 (1977).

${ }^{22}$ L. D. Landau, E. M. Lifshitz, and L. P. Pitaevskii, Electrodynamics of Continuous Media, 2nd ed. (Pergamon, Oxford, 1984).

${ }^{23}$ W. H. Press, B. P. Flannery, S. A. Teukolsky, and W. T. Vetterling, $\mathrm{Nu}-$ merical Recipes: The Art of Scientific Computing (Cambridge University Press, Cambridge, 1986). 